\title{
Homogeneous quaternionic Kähler structures on rank-three Alekseevsky spaces
}

\author{
WafaA Batat, Pedro M. Gadea, And José A. Oubiña *
}

\begin{abstract}
The homogeneous quaternionic Kähler structures on the rank-three Alekseevsky spaces with their natural quaternionic structures, when each of them is described as a solvable Lie group, and their types in Fino's classification, are found.
\end{abstract}

Keywords: Alekseevsky spaces, Quaternion-Kähler manifolds, Homogeneous quaternionic Kähler structures.

A.M.S. Subject Classification 2010: 53C26, 53C30.

\section{Introduction}

As it is well known, symmetric quaternion-Kähler spaces were classified by Wolf [21] and homogeneous quaternion-Kähler spaces admitting a simply transitive real solvable group of isometries were classified by Alekseevsky [2] (see also de Wit and van Proeyen [20] and Cortés [11]). No more homogeneous quaternionKähler spaces are known. Alekseevsky proved in [1] that compact homogeneous quaternion-Kähler manifolds are Wolf spaces, and conjectured in [2, p. 300] that the only homogeneous quaternion-Kähler manifolds are either Wolf spaces or Alekseevsky spaces. A quaternion-Kähler manifold is said to be positive (resp. negative) if it is complete and has positive (resp. negative) scalar curvature. LeBrun and Salamon's Conjecture [16] says that a positive quaternion-Kähler manifold must be isometric to a Wolf space. This was settled by Hitchin [13] and Poon and Salamon [17] for dimensions four and eight, respectively.

In turn, homogeneous quaternionic Kähler structures, that is, the $\operatorname{Sp}(n) \operatorname{Sp}(1)$ case of the Ambrose and Singer [4] and Tricerri and Vanhecke [19] homogeneous Riemannian structures, have been studied in [12], [9], [7], [8]. Fino gave in [12] a representation-theoretical classification of homogeneous quaternionic Kähler structures, which has five basic geometric types $\mathcal{Q} \mathcal{K}_{1}, \ldots, \mathcal{Q} \mathcal{K}_{5}$. (In the sequel we shall denote simply the type $\mathcal{Q} \mathcal{K}_{i} \oplus \mathcal{Q K}{ }_{j}$ by $\mathcal{Q K}{ }_{i j}$, the type $\mathcal{Q} \mathcal{K}_{i} \oplus \mathcal{Q K}{ }_{j} \oplus \mathcal{Q} \mathcal{K}_{k}$ by $\mathcal{Q} \mathcal{K}_{i j k}$, and so on.) A classification of these structures in terms of real tensors

${ }^{*}$ The first author has been partially supported by the ENSET d'Oran, Algeria. The second and third authors have been partially supported by the Ministry of Science and Innovation, Spain, under Project MTM2008-01386. 
was then given in [9] (see Theorem 2.1 below), where it was moreover proved that a connected, simply-connected and complete homogeneous quaternion-Kähler manifold of real dimension equal or greater than eight, admitting a nonvanishing structure in $\mathcal{Q} \mathcal{K}_{123}$ with nonzero projection to $\mathcal{Q} \mathcal{K}_{3}$, is necessarily the quaternionic hyperbolic space $\mathbb{H} H(n)$. Furthermore, a structure of type $\mathcal{Q} \mathcal{K}_{134}$ on $\mathbb{H H}(n)$, corresponding to its description as a solvable group, has been given in [9]. Then, in [7], [8] it has been shown that the quaternion-Kähler symmetric spaces of dimension either 8 or 12 (they also are Alekseevsky spaces) furnish proper realisations of the types $\mathcal{Q} \mathcal{K}_{134}, \mathcal{Q} \mathcal{K}_{135}, \mathcal{Q K} \mathcal{K}_{1345}$ and the generic type $\mathcal{Q K}_{12345}$.

On the other hand, quaternion-Kähler spaces appear in the formulation of the coupling of matter fields in $N=2$ supergravity. Each multiplet consists of $4 n$ real scalars and two Majorana spinor fields. The $4 n$ real scalars parameterise a $4 n$-dimensional Riemannian manifold $M$. If the gravity is considered as a dynamical field, the holonomy group of $M$ is a subgroup of $\operatorname{Sp}(n) \operatorname{Sp}(1)$, and $M$ is a negative quaternion-Kähler manifold (Bagger and Witten [5], see also [10], [20]), so these spaces are target spaces of nonlinear $\sigma$-models in $N=2, d=4$, supergravity. Later, Cecotti [10] studied the relation of those spaces with the $c$ map, and proved that Alekseevsky spaces naturally appear in this context and, furthermore, that the nonsymmetric ones are related to Vinberg $T$-algebras as the symmetric ones are related to Jordan algebras. In turn, de Wit and Van Proeyen [20] studied those spaces, completing Alekseevsky's classification, and specifically adding the spaces $\mathcal{T}(p), p \geq 1$, by using supergravity considerations. That Alekseevsky spaces actually appear in three series, $\mathcal{T}$-, $\mathcal{W}$-, and $\mathcal{V}$-spaces (which include symmetric and nonsymmetric manifolds), was then proved by Cortés [11] by using geometric arguments. Further, the authors of [20] gave an explanation of the presence of nonsymmetric spaces in terms of (certain extra) dimensions of the isometry groups.

The aim of the present paper is to find the explicit homogeneous quaternionic Kähler structures carried by the rank-three Alekseevsky spaces $\mathcal{T}(p), p \geq 0$, when each of them is described as a solvable Lie group, and then to determine the type of such structures in Fino's classification, so seeing in particular whether the structure is or not special, in the sense of having or not nonzero components in each basic type $\mathcal{Q} \mathcal{K}_{i}, i=1, \ldots, 5$.

As for the contents, after some preliminaries in $\S 2$, we obtain in $\S 3$ Theorem 3.1 , giving the homogeneous quaternionic Kähler structure corresponding to the description as a Lie group of each space $\mathcal{T}(p)$. In $\S 4$ we give Theorem 4.1, with the type of such structure in Fino's classification. We prove that it has components in each subspace $\mathcal{Q} \mathcal{K}_{i}$, for $i=1, \ldots, 5$. 


\section{Preliminaries}

\subsection{Homogeneous quaternionic Kähler structures}

As it is well known, Ambrose and Singer [4] proved that a connected, simplyconnected and complete Riemannian manifold $(M, g)$ is Riemannian homogeneous if and only if it admits a homogeneous Riemannian structure, i.e., a $(1,2)$ tensor field $S$ satisfying

$$
\widetilde{\nabla} g=0, \quad \widetilde{\nabla} R=0, \quad \widetilde{\nabla} S=0,
$$

where $\widetilde{\nabla}=\nabla-S, \nabla$ denotes the Levi-Civita connection and $R$ the curvature tensor of $\nabla$. We write as usual $S_{X Y Z}=g\left(S_{X} Y, Z\right)$. Then, from $\nabla g=0$ it follows that the condition $\widetilde{\nabla} g=0$ is equivalent to $S_{X Z Y}=-S_{X Y Z}$.

Let $\left(M, g, v^{3}\right)$ be an almost quaternion-Hermitian manifold, $v^{3}$ being the structure subbundle of the bundle of $(1,1)$ tensors on $M$. That is, there locally exists a basis $J_{1}, J_{2}, J_{3}$ of $v^{3}$ satisfying the conditions

$$
J_{a}^{2}=-I, \quad J_{1} J_{2}=-J_{2} J_{1}=J_{3}, \quad g\left(J_{a} X, J_{a} Y\right)=g(X, Y),
$$

for $a=1,2,3$. Such a basis is called a standard local basis of $v^{3}$ in its domain of definition. Such an $M$ has dimension $4 n$, with $n \geq 1$. It is known that $M$ admits an almost quaternion-Hermitian structure if and only if the structure group of its tangent bundle $T M$ is reducible to $\operatorname{Sp}(n) \operatorname{Sp}(1)$.

Let $J_{1}, J_{2}, J_{3}$ be a standard local basis of $v^{3}$ and let $\omega_{a}(X, Y)=g\left(J_{a} X, Y\right)$, $a=1,2,3$. The differential 4-form $\Omega=\sum_{a=1}^{3} \omega_{a} \wedge \omega_{a}$ is known to be globally defined. The manifold is said to be quaternion-Kähler if one has locally (cf. Ishihara [14]) that

$$
\nabla_{X} J_{1}=\tau^{3}(X) J_{2}-\tau^{2}(X) J_{3}, \quad \text { etc. }
$$

for certain differential 1-forms $\tau^{1}, \tau^{2}, \tau^{3}$ (here and in the sequel, 'etc.' denotes the equations obtained by cyclically permuting the indices $1,2,3)$; or, equivalently, if $\nabla \Omega=0$. The holonomy group is contained in $\operatorname{Sp}(n) \operatorname{Sp}(1)$.

In the present paper we shall consider quaternion-Kähler manifolds of $\operatorname{dim} \geq$ 8 and negative scalar curvature (see [6], [18]). We have (cf. Alekseevsky and Cortés [3, p. 218], and see also [9, Rem. 2.2]) the next

Definition 2.1. A quaternion-Kähler manifold $\left(M, g, v^{3}\right)$ is said to be a homogeneous quaternion-Kähler manifold if it admits a transitive group of isometries.

Moreover, as a Corollary to Kiričenco's Theorem [15] one has that a connected, simply-connected and complete quaternion-Kähler manifold $\left(M, g, v^{3}\right)$ is homogeneous if and only if there exists a tensor field $S$ of type $(1,2)$ on $M$ satisfying

$$
\widetilde{\nabla} g=0, \quad \widetilde{\nabla} R=0, \quad \widetilde{\nabla} S=0, \quad \widetilde{\nabla} \Omega=0,
$$

where $\widetilde{\nabla}=\nabla-S$. Such a tensor $S$ is called a homogeneous quaternionic Kähler structure on $M$. The equation $\widetilde{\nabla} \Omega=0$ is equivalent, under $\widetilde{\nabla} g=0$, to the 
existence of three differential 1-forms $\tilde{\tau}^{1}, \tilde{\tau}^{2}, \tilde{\tau}^{3}$ such that

$$
\widetilde{\nabla}_{X} J_{1}=\tilde{\tau}^{3}(X) J_{2}-\tilde{\tau}^{2}(X) J_{3}, \quad \text { etc. }
$$

Formulas (2.2) and (2.4) yield

$$
S_{X} J_{1} Y-J_{1} S_{X} Y=\theta^{3}(X) J_{2} Y-\theta^{2}(X) J_{3} Y, \quad \text { etc. }
$$

for $\theta^{a}=\tau^{a}-\tilde{\tau}^{a}, a=1,2,3$. We then have that

$$
S_{X J_{1} Y J_{1} Z}-S_{X Y Z}=\theta^{3}(X) g\left(J_{2} Y, J_{1} Z\right)-\theta^{2}(X) g\left(J_{3} Y, J_{1} Z\right), \quad \text { etc., }
$$

which together with the condition $S_{X Y Z}=-S_{X Z Y}$, are the symmetries satisfied by a homogeneous quaternionic Kähler structure $S$.

Fino gave a representation-theoretical classification of homogeneous quaternionic Kähler structures: With the usual notation $E, H$ (and $K$ for the module with highest weight $(2,1,0, \ldots, 0))$ for quaternion-Kähler manifolds (see for instance [18]) one has

Theorem (Fino [12, Lemma 5.1]) A homogeneous quaternionic Kähler structure $S$ belongs pointwise to the module

$$
[E H] \otimes(\mathfrak{s p}(1) \oplus \mathfrak{s p}(n)) \cong[E H]+\left[E S^{3} H\right]+[E H]+\left[S^{3} E H\right]+[K H] .
$$

We shall write $\mathcal{Q} \mathcal{K}_{1}, \ldots, \mathcal{Q} \mathcal{K}_{5}$ for the five Fino basic types, in the above order.

Let $(V,\langle\rangle,, \mathrm{q})$ be a quaternion-Hermitian vector space, i.e., a $4 n$-dimensional real vector space endowed with an inner product $\langle$,$\rangle and a quaternionic struc-$ ture q generated by operators $J_{1}, J_{2}, J_{3}$ satisfying (2.1). Consider the space of tensors

$$
\mathcal{T}(V)=\left\{S \in \otimes^{3} V^{*}: S_{X Y Z}=-S_{X Z Y}\right\}
$$

and its vector subspace

$$
\mathcal{Q K}(V)=\left\{S \in \otimes^{3} V^{*}: S_{X Y Z}=-S_{X Z Y}, \exists \theta^{a} \in V^{*} \text { s.t. } S \text { satisfies (2.5) }\right\} .
$$

Any homogeneous Riemannian structure on $M$ belongs to $\mathcal{T}\left(T_{p} M\right)$ pointwise, but homogeneous quaternionic Kähler structures are pointwise in $\mathcal{Q K}\left(T_{p} M\right)$.

Consider the subspaces $\check{\mathcal{V}}$ and $\widehat{\mathcal{V}}$ of $\mathcal{Q K}(V)$ defined by

$$
\begin{gathered}
\check{\mathcal{V}}=\left\{\Theta \in \mathcal{Q K}(V): \Theta_{X Y Z}=\sum_{a=1}^{3} \theta^{a}(X)\left\langle J_{a} Y, Z\right\rangle, \theta^{a} \in V^{*}\right\}, \\
\widehat{\mathcal{V}}=\left\{\mathcal{A} \in \mathcal{Q K}(V): \mathcal{A}_{X J_{a} Y J_{a} Z}=\mathcal{A}_{X Y Z}, a=1,2,3\right\} .
\end{gathered}
$$

Then one has $\mathcal{Q} \mathcal{K}(V)=\check{\mathcal{V}} \oplus \widehat{\mathcal{V}}$, and each element $S \in \mathcal{Q K}(V)$ decomposes as

$$
S_{X Y Z}=\Theta_{X Y Z}+\mathcal{A}_{X Y Z}
$$


where

$$
\Theta_{X Y Z}=\frac{1}{2} \sum_{a=1}^{3} \theta^{a}(X)\left\langle J_{a} Y, Z\right\rangle,
$$

$\theta^{a}$ being the 1 -forms corresponding to $S$ as in (2.5). Moreover, the classification by real tensors is $([9$, Th. 3.15]) as follows.

Theorem 2.1. If $n \geq 2$, the space $\mathcal{Q K}(V)$ decomposes into the direct sum of the following $\operatorname{Sp}(n) \operatorname{Sp}(1)$-invariant and irreducible subspaces:

$$
\begin{aligned}
& \mathcal{Q} \mathcal{K}_{1}=\left\{\Theta \in \check{\mathcal{V}}: \Theta_{X Y Z}=\sum_{a=1}^{3} \theta\left(J_{a} X\right)\left\langle J_{a} Y, Z\right\rangle, \theta \in V^{*}\right\}, \\
& \mathcal{Q} \mathcal{K}_{2}=\left\{\Theta \in \check{\mathcal{V}}: \Theta_{X Y Z}=\sum_{a=1}^{3} \theta^{a}(X)\left\langle J_{a} Y, Z\right\rangle, \sum_{a=1}^{3} \theta^{a} \circ J_{a}=0, \theta^{a} \in V^{*}\right\}, \\
& \mathcal{Q K}_{3}=\left\{\mathcal{A} \in \widehat{\mathcal{V}}: \mathcal{A}_{X Y Z}=\langle X, Y\rangle \vartheta(Z)-\langle X, Z\rangle \vartheta(Y)\right. \\
& \left.+\sum_{a=1}^{3}\left(\left\langle X, J_{a} Y\right\rangle \vartheta\left(J_{a} Z\right)-\left\langle X, J_{a} Z\right\rangle \vartheta\left(J_{a} Y\right)\right), \vartheta \in V^{*}\right\} \\
& \mathcal{Q} \mathcal{K}_{4}=\left\{\mathcal{A} \in \widehat{\mathcal{V}}: \mathcal{A}_{X Y Z}=\frac{1}{6}\left(\mathfrak{S}_{X Y Z} \mathcal{A}_{X Y Z}+\sum_{a=1}^{3} \underset{X J_{a} Y J_{a} Z}{\mathfrak{S}} \mathcal{A}_{X J_{a} Y J_{a} Z}\right),\right. \\
& \left.c_{12}(\mathcal{A})=0\right\} \\
& \mathcal{Q} \mathcal{K}_{5}=\left\{\mathcal{A} \in \widehat{\mathcal{V}}: \underset{X Y Z}{\mathfrak{S}} \mathcal{A}_{X Y Z}=0\right\},
\end{aligned}
$$

where $c_{12}(\mathcal{A})(Z)=\sum_{i=1}^{4 n} \mathcal{A}_{e_{i} e_{i} Z}$, for any local orthonormal basis $\left\{e_{i}\right\}$ of $V$.

Note that with the previous notations we have that

$$
\check{\mathcal{V}}=\mathcal{Q} \mathcal{K}_{12}, \quad \widehat{\mathcal{V}}=\mathcal{Q K}_{345}
$$

\subsection{Alekseevsky spaces}

We recall here some definitions and results by Alekseevsky [2] (see also [3], [11]). A solvable Lie algebra $\mathfrak{s}$ is said to be real (or completely) solvable, if the endomorphisms $\operatorname{ad}_{X}, X \in \mathfrak{s}$, have only real eigenvalues. A Lie group is said to be real solvable if its Lie algebra is real solvable. A quaternion-Kähler manifold of nonzero scalar curvature is said to be an Alekseevsky space if it admits a simply transitive, real solvable group of isometries.

A metric Lie algebra is a pair $(\mathfrak{s},\langle\rangle$,$) consisting of a Lie algebra \mathfrak{s}$ and an inner product $\langle$,$\rangle on \mathfrak{s}$. An Alekseevsky space is simply-connected and it can be regarded as a real solvable Lie group with a left-invariant metric. The corresponding metric Lie algebra with the quaternionic structure inherited from 
that of the manifold is a quaternion-Hermitian vector space $(\mathfrak{s},\langle\rangle, q$,$) which is$ called a quaternionic or Alekseevskian Lie algebra. A metric Lie algebra $\mathfrak{f}$ with an orthonormal basis $\{G, H\}$ and complex structure $J$ is said to be a key algebra with root $\mu$, if

$$
G=J H, \quad[H, G]=\mu G, \quad \mu>0 .
$$

A metric Lie algebra $\mathfrak{f}+\mathfrak{x}$ with complex structure $J$ is said to be an elementary Kählerian Lie algebra with root $\mu$ if $\mathfrak{f}=\operatorname{Span}\{G, H\}$ is a key subalgebra with root $\mu$ and

$$
\left.\operatorname{ad}_{H}\right|_{\mathfrak{x}}=\frac{1}{2} \mu I,\left.\quad \operatorname{ad}_{G}\right|_{\mathfrak{x}}=0, \quad[X, Y]=\mu\langle J X, Y\rangle G, \quad X, Y \in \mathfrak{x} .
$$

A representation $U \mapsto T_{U}$ of a Lie algebra $\mathfrak{u}$ with complex structure $J$ on a Euclidean space $(\mathfrak{x},\langle\rangle$,$) with a complex structure J_{1}$ is said to be symplectic if:

(1) $J_{1} T_{U} J_{1}=T_{U}^{\prime}$ (where $T_{U}^{\prime}$ is the endomorphism of $\mathfrak{x}$ adjoint to $T_{U}$ ), i.e., $T_{U}$ annihilates the Kähler form $\omega_{1}=\left\langle J_{1} \cdot, \cdot\right\rangle: \omega_{1}\left(T_{U} X, Y\right)+\omega_{1}\left(X, T_{U} Y\right)=0$ for all $U \in \mathfrak{u}, X, Y \in \mathfrak{x}$.

(2) $T_{J U}^{\mathrm{sym}}=J_{1} T_{U}^{\mathrm{sym}}$ for all $U \in \mathfrak{u}$, where $T_{U}^{\mathrm{sym}}=\frac{1}{2}\left(T_{U}+T_{U}^{\prime}\right)$ denotes the symmetric part of $T_{U}$.

If $T_{\mathfrak{u}} \mathfrak{x}=\mathfrak{x}, T$ is called nondegenerate. If $T$ is a nondegenerate symplectic representation of a key algebra $\mathfrak{f}=\operatorname{Span}\{G, H\}$ with root $\mu$ on a space $\left(\mathfrak{x},\langle\rangle,, J_{1}\right)$, then $\mathfrak{x}$ admits a weight decomposition $\mathfrak{x}=\mathfrak{x}_{+}+\mathfrak{x}_{-}$such that

$$
\mathfrak{x}_{-}=J_{1} \mathfrak{x}_{+},\left.\quad T_{G}\right|_{\mathfrak{x}_{+}}=0,\left.\quad T_{G}\right|_{\mathfrak{x}_{-}}=-\mu J_{1},\left.\quad T_{H}\right|_{\mathfrak{x}_{ \pm}}= \pm \frac{1}{2} \mu I .
$$

Any Alekseevskian algebra $(\mathfrak{s},\langle\rangle,, \mathrm{q})$, with $\mathrm{q}=\operatorname{Span}\left\{J_{a}: a=1,2,3\right\}$, contains one (up to scaling) 1-dimensional quaternionic subalgebra $\mathfrak{s}^{\prime}$ (i.e., a subalgebra $\mathfrak{s}^{\prime}$ such that $\left.q \mathfrak{s}^{\prime} \subset \mathfrak{s}^{\prime}\right)$, corresponding either to the complex hyperbolic plane $\mathbb{C H}(2)$ or to the quaternionic hyperbolic line $\mathbb{H} H(1)$. In the former case it is of the form $\mathfrak{s}=\mathfrak{u}+J_{2} \mathfrak{u}$ (orthogonal sum), and $\left(\mathfrak{u}, J_{1 \mid \mathfrak{u}}\right)$ is the socalled principal Kählerian subalgebra of $\mathfrak{s}$. The Lie algebra $\mathfrak{u}$ contains a key subalgebra $\mathfrak{f}_{0}=\operatorname{Span}\left\{G_{0}, H_{0}\right\}$ with root 1 such that $\mathfrak{f}_{0}+J_{2} \mathfrak{f}_{0}$ is the canonical 1-dimensional quaternionic subalgebra of $\mathfrak{s}$, and the adjoint representation of $\mathfrak{s}$ induces a representation of $\mathfrak{u}$ on $\mathfrak{u}^{\perp}=J_{2} \mathfrak{u}$, which furnishes the model for the next definition.

A Kählerian Lie algebra $(\mathfrak{u}, J)$, that is, a metric Lie algebra which corresponds to a Kählerian homogeneous space, is said to be admissible if $\mathfrak{u}=\mathfrak{f}_{0}+\mathfrak{u}_{0}$ is a direct orthogonal sum of a key algebra $\mathfrak{f}_{0}=\operatorname{Span}\left\{G_{0}, H_{0}\right\}$ with root 1 and a real solvable Kählerian Lie algebra $\mathfrak{u}_{0}$. A representation $U \mapsto T_{U}$ of such a Lie algebra $\mathfrak{u}$ on a Euclidean space $\tilde{\mathfrak{u}}$ together with a vector space isometry $\varphi: \mathfrak{u} \rightarrow \tilde{\mathfrak{u}}$ is said to be a Q-representation if it satisfies the following eight conditions (Q1-8), given in Alekseevsky [2, Lemma 5.5 and Def. 5.3] (cf. also Cortés $\left[11\right.$, Def. 1.8]), where we denote $\widetilde{U}=\varphi(U)$ for each $U \in \mathfrak{u}$, and $J_{1}$ and $\hat{J}$ are the complex structures on $\tilde{\mathfrak{u}}$ given by

$$
J_{1}=-\varphi J \varphi^{-1}, \quad \hat{J}\left|\widetilde{\mathfrak{f}}_{0}=-J_{1}\right| \widetilde{\mathfrak{f}}_{0}, \quad \hat{J}\left|\widetilde{\mathfrak{u}}_{0}=J_{1}\right| \widetilde{\mathfrak{u}}_{0}:
$$

(Q1) $T_{H_{0}}=\frac{1}{2} I, \quad T_{G_{0}}=0$, 
(Q2) $T_{U_{0}} \widetilde{H}_{0}=\frac{1}{2} \widetilde{U}_{0}-2 \alpha\left(U_{0}\right) \widetilde{G}_{0}, \quad T_{U_{0}} \widetilde{G}_{0}=\frac{1}{2} \widetilde{J U} \widetilde{U}_{0}+2 \alpha\left(U_{0}\right) \widetilde{H}_{0}$,

(Q3) $T_{U_{0}} \widetilde{V}_{0} \equiv \frac{1}{2}\left\langle U_{0}, V_{0}\right\rangle \widetilde{H}_{0}+\frac{1}{2}\left\langle J U_{0}, V_{0}\right\rangle \widetilde{G}_{0} \bmod \tilde{\mathfrak{u}}_{0}$

(Q4) $T_{U_{0}}^{\mathrm{sk}}=\varphi \nabla_{U_{0}} \varphi^{-1}+2 \alpha\left(U_{0}\right) J_{1}$,

(Q5) $\left.T_{J U_{0}}^{\mathrm{sym}}\right|_{\tilde{\mathfrak{u}}_{0}}=\left.J_{1} T_{U_{0}}^{\mathrm{sym}}\right|_{\tilde{\mathfrak{u}}_{0}}$,

(Q6) $T_{U_{0}}^{\mathrm{sym}} \widetilde{V}_{0}-T_{V_{0}}^{\mathrm{sym}} \widetilde{U}_{0}=\left\langle J U_{0}, V_{0}\right\rangle \widetilde{G}_{0}$,

(Q7) $T_{U_{0}}$ annihilates the Kähler form $\hat{\omega}=\langle\hat{J} \cdot, \cdot\rangle$,

for some linear form $\alpha$ on $\mathfrak{u}_{0}$, where $U_{0}, V_{0} \in \mathfrak{u}_{0}, \nabla$ is the covariant derivative of $\mathfrak{u}$, and $T_{U_{0}}^{\text {sk }}$ and $T_{U_{0}}^{\text {sym }}$ denote the skew and symmetric parts of $T_{U_{0}}$, respectively; and

(Q8) for each $U \in \mathfrak{u}$, the endomorphism $T_{U}$ has only real eigenvalues.

Then Alekseevsky proved in [2] the following facts: Q-representations of an admissible Kählerian Lie algebra $(\mathfrak{u}, J)$ and Alekseevskian Lie algebras with principal Kählerian subalgebra $(\mathfrak{u}, J)$ are related by the following natural correspondence. If $\mathfrak{s}$ is an Alekseevskian Lie algebra with principal Kählerian subalgebra $(\mathfrak{u}, J)$, then the representation of $\mathfrak{u}$ on $J_{2} \mathfrak{u}$ induced by the adjoint representation of $\mathfrak{s}$ is a Q-representation with $\varphi=\left.J_{2}\right|_{\mathfrak{u}}: \mathfrak{u} \rightarrow \mathfrak{u}^{\perp}$. Conversely, let $(T, \varphi)$ be a Q-representation of an admissible Kählerian Lie algebra $(\mathfrak{u}, J)$ on the Euclidean vector space

$$
\tilde{\mathfrak{u}}=\varphi(\mathfrak{u})=\tilde{\mathfrak{f}}_{0}+\widetilde{\mathfrak{u}}_{0} .
$$

A quaternionic structure $\mathrm{q}=\operatorname{Span}\left\{J_{a}: a=1,2,3\right\}$ on the Euclidean vector space $\mathfrak{s}=\mathfrak{u}+\tilde{\mathfrak{u}}$ (orthogonal sum) is defined by

$$
\left.J_{1}\right|_{\mathfrak{u}}=J,\left.\quad J_{1}\right|_{\mathfrak{u}}=-\varphi J \varphi^{-1},\left.\quad J_{2}\right|_{\mathfrak{u}}=\varphi,\left.\quad J_{2}\right|_{\tilde{\mathfrak{u}}}=-\varphi^{-1}, \quad J_{3}=J_{1} J_{2} .
$$

Let $\hat{J}$ be the complex structure on $\tilde{\mathfrak{u}}$ defined as in (2.9), and let $\hat{\omega}$ denote the Kähler form on $\tilde{\mathfrak{u}}$ given by $\hat{\omega}(\widetilde{U}, \widetilde{V})=\langle\hat{J} \widetilde{U}, \widetilde{V}\rangle$. Then the following conditions define the structure of Lie algebra of $\mathfrak{s}$ :

$\mathfrak{u}$ is a subalgebra of $\mathfrak{s},\left.\quad \operatorname{ad}_{U}\right|_{\tilde{\mathfrak{u}}}=T_{U}, \quad[\widetilde{U}, \tilde{V}]=\hat{\omega}(\widetilde{U}, \widetilde{V}) G_{0}$,

for all $U, V \in \mathfrak{u}$.

The rank of a solvable Lie algebra $\mathfrak{s}$ is the dimension of a Cartan subalgebra of $\mathfrak{s}$. The rank of an Alekseevsky space $\mathcal{S}$ is the rank of its Alekseevskian Lie algebra $\mathfrak{s}$, which is proved to be at most 4 . An admissible Kählerian Lie algebra $\mathfrak{u}=\mathfrak{f}_{0}+\mathfrak{u}_{0}$ which admits a Q-representation decomposes as a semidirect sum of elementary Kählerian Lie algebras, with $\mathfrak{u}_{0}=\sum_{i \geq 1}\left(\mathfrak{f}_{i}+\mathfrak{x}_{i}\right)$, that is,

$$
\left[\mathfrak{f}_{i}+\mathfrak{x}_{i}, \mathfrak{f}_{j}+\mathfrak{x}_{j}\right] \subset \mathfrak{f}_{j}+\mathfrak{x}_{j}, \quad i \geq j
$$

with symplectic representation $\left.\operatorname{ad}_{\mathfrak{f}_{i}}\right|_{\mathfrak{x}_{j}}$ for $i>j$ and commuting key algebras, $\left[\mathfrak{f}_{i}, \mathfrak{f}_{j}\right]=0$, for $i \neq j$ (see [11, p. 134]).

The rank of $\mathfrak{u}=\mathfrak{f}_{0}+\sum_{i>1}\left(\mathfrak{f}_{i}+\mathfrak{x}_{i}\right)$ coincides with the number of the key algebras of $\mathfrak{u}$. There are only three types (type 1, 2 or 3 ) of admissible Kählerian Lie algebras, corresponding to the cases where the smallest root is $1,1 / \sqrt{2}$ or $1 / \sqrt{3}$, respectively. 


\section{Homogeneous quaternionic Kähler structures on the spaces $\mathcal{T}(p)$}

Kählerian Lie algebras of type 2 which admit a Q-representation have rank 3. Now, (see [2, Sect. 8] and [11, Sect. 2.1]) every Kählerian Lie algebra (u, $J$ ) of type 2 which admits a Q-representation is a direct (orthogonal) sum of the form $\mathfrak{u}=\mathfrak{f}_{0}+\mathfrak{u}_{0}$, where $\mathfrak{f}_{0}=\operatorname{Span}\left\{G_{0}, H_{0}\right\}$ is a key algebra with root 1 and $\mathfrak{u}_{0}$ is a semidirect sum of two elementary Kählerian Lie algebras $\mathfrak{f}_{1}+\mathfrak{x}_{1}$ and $\mathfrak{f}_{2}$ with roots 1 and $1 / \sqrt{2}$, respectively, the key algebras $\mathfrak{f}_{i}=\operatorname{Span}\left\{G_{i}, H_{i}\right\}, i=1,2$, commute and $\left.\operatorname{ad}_{\mathfrak{f}_{2}}\right|_{\mathfrak{x}_{1}}$ is a nondegenerate symplectic representation with weight decomposition

$$
\mathfrak{x}_{1}=\mathfrak{x}_{+}+\mathfrak{x}_{-}, \quad \mathfrak{x}_{-}=J \mathfrak{x}_{+} .
$$

Then, by (2.7) and (2.8) respectively, we have

$$
\left.\operatorname{ad}_{H_{1}}\right|_{\mathfrak{x}_{1}}=\frac{1}{2} I,\left.\quad \operatorname{ad}_{G_{1}}\right|_{\mathfrak{x}_{1}}=0, \quad[X, Y]=\langle J X, Y\rangle G_{1}, \quad X, Y \in \mathfrak{x}_{1},
$$

and

$$
\left.\operatorname{ad}_{G_{2}}\right|_{\mathfrak{x}_{+}}=0,\left.\quad \operatorname{ad}_{G_{2}}\right|_{\mathfrak{x}_{-}}=-\frac{\sqrt{2}}{2} J,\left.\quad \operatorname{ad}_{H_{2}}\right|_{\mathfrak{x}_{ \pm}}= \pm \frac{\sqrt{2}}{4} I .
$$

Thus we have the following Lie brackets on $\mathfrak{u}$ (where we put $X_{-}=J X_{+} \in \mathfrak{x}_{-}$ for each $X_{+} \in \mathfrak{x}_{+}$):

$$
\begin{aligned}
& {\left[H_{0}, G_{0}\right]=G_{0}, \quad\left[H_{1}, G_{1}\right]=G_{1}, \quad\left[H_{2}, G_{2}\right]=\frac{\sqrt{2}}{2} G_{2},} \\
& {\left[H_{1}, X\right]=\frac{1}{2} X, \quad\left[G_{1}, X\right]=0, \quad \text { for } X \in \mathfrak{x}_{1},} \\
& {\left[X_{+}, Y_{+}\right]=\left[X_{-}, Y_{-}\right]=0, \quad\left[X_{+}, Y_{-}\right]=\left\langle X_{+}, Y_{+}\right\rangle G_{1},} \\
& {\left[G_{2}, X_{+}\right]=0, \quad\left[G_{2}, X_{-}\right]=\frac{\sqrt{2}}{2} X_{+},} \\
& {\left[H_{2}, X_{+}\right]=\frac{\sqrt{2}}{4} X_{+}, \quad\left[H_{2}, X_{-}\right]=-\frac{\sqrt{2}}{4} X_{-},}
\end{aligned}
$$

for all $X_{+}, Y_{+} \in \mathfrak{x}_{+}$. These, together with $\left[\mathfrak{f}_{0}, \mathfrak{u}_{0}\right]=0$ and $\left[\mathfrak{f}_{1}, \mathfrak{f}_{2}\right]=0$, give the structure of Lie algebra of $\mathfrak{u}$.

Furthermore, the Kählerian Lie algebra $(\mathfrak{u}, J)$ has a unique Q-representation on the Euclidean vector space $\tilde{\mathfrak{u}}=\tilde{\mathfrak{f}}_{0}+\widetilde{\mathfrak{u}}_{0}$,

$$
T: \mathfrak{u} \rightarrow \operatorname{End}(\tilde{\mathfrak{u}}),
$$

where $\sim: \mathfrak{u} \rightarrow \tilde{\mathfrak{u}}$ denotes the corresponding isometry of Euclidean vector spaces.

If $p=\operatorname{dim}_{\mathbb{C}} \mathfrak{x}_{1}=\operatorname{dim}_{\mathbb{R}} \mathfrak{x}_{+} \in\{0,1,2, \ldots\}$, we consider the quaternionHermitian vector space $(\mathfrak{t}(p),\langle\rangle,, \mathrm{q})$, where $\mathfrak{t}(p)=\mathfrak{u}+\tilde{\mathfrak{u}}$ is a direct orthogonal sum, and $\mathrm{q}=\operatorname{Span}\left\{J_{a}: a=1,2,3\right\}$ is the quaternionic structure on $\mathfrak{t}(p)$ defined by $(2.10)$. Then

Let $\left\{X_{i+}: 1 \leq i \leq p\right\}$ be an orthonormal basis of $\mathfrak{x}_{+}$, and $X_{i-}=J X_{i+}$.

$$
\mathcal{B}=\left\{G_{j}, H_{j}, X_{i+}, X_{i-}, \widetilde{G}_{j}, \widetilde{H}_{j}, \widetilde{X}_{i_{+}}, \widetilde{X}_{i_{-}}: 0 \leq j \leq 2,1 \leq i \leq p\right\}
$$


Table 1: The action of $J_{a}, a=1,2,3$, on $\mathfrak{t}(p)$

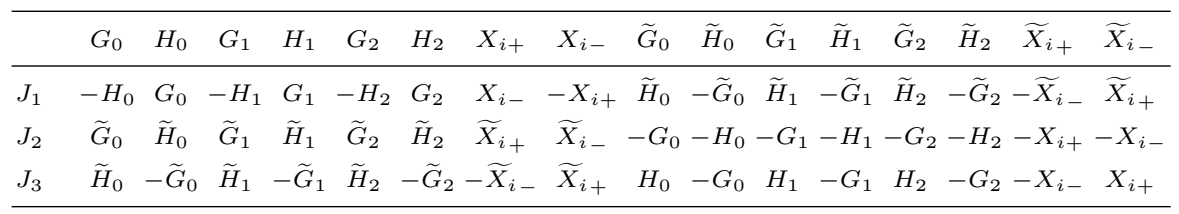

is an orthonormal basis of $\mathfrak{t}(p)$.

The action of $J_{a}, a=1,2,3$, on $\mathfrak{t}(p)=\mathfrak{u}+\tilde{\mathfrak{u}}$ is given in Table 1 .

The vector space $\mathfrak{t}(p)$ has a structure of Lie algebra given by (2.11), with $\mathfrak{s}=\mathfrak{t}(p)$, where the complex structure $\hat{J}$ on $\tilde{\mathfrak{u}}$ is defined by

\begin{tabular}{ccccccccc}
\hline & $\widetilde{G}_{0}$ & $\widetilde{H}_{0}$ & $\widetilde{G}_{1}$ & $\widetilde{H}_{1}$ & $\widetilde{G}_{2}$ & $\widetilde{H}_{2}$ & $\widetilde{X}_{i+}$ & $\widetilde{X}_{i-}$ \\
\hline$\hat{J}$ & $-\widetilde{H}_{0}$ & $\widetilde{G}_{0}$ & $\widetilde{H}_{1}$ & $-\widetilde{G}_{1}$ & $\widetilde{H}_{2}$ & $-\widetilde{G}_{2}$ & $-\widetilde{X}_{i-}$ & $\widetilde{X}_{i+}$ \\
\hline
\end{tabular}

Then, by the third condition in (2.11), we have the brackets of the elements of $\tilde{u}$. The nonnull brackets are given by

$$
\left[\widetilde{H}_{0}, \widetilde{G}_{0}\right]=-\left[\widetilde{H}_{1}, \widetilde{G}_{1}\right]=-\left[\widetilde{H}_{2}, \widetilde{G}_{2}\right]=-\left[\widetilde{X}_{i+}, \widetilde{X}_{i-}\right]=G_{0} .
$$

If $U \in \mathfrak{u}$ and $\tilde{V} \in \tilde{\mathfrak{u}}$, by the second condition in (2.11), $[U, \tilde{V}]=T_{U} \tilde{V}$, and it is exhibited in Table 2, where $T: \mathfrak{u} \rightarrow \operatorname{End}(\tilde{\mathfrak{u}})$ is expressed in terms of the orthonormal basis $\left\{\widetilde{G}_{j}, \widetilde{H}_{j}, \widetilde{X}_{i+}, \widetilde{X}_{i-}\right\}$ of $\tilde{\mathfrak{u}}$, from the conditions (Q1-8) of a Q-representation (cf. Cortés [11, Prop. 2.1]).

The Lie algebra $\mathfrak{t}(p)$ is 4 -step solvable, $\operatorname{dim} \mathfrak{t}(p)=12+4 p$, and the corresponding simply-connected Lie group with left-invariant metric is the Alekseevsky space $\mathcal{T}(p)$.

Consider now, for any $p \geq 0$, the Alekseevsky space $\mathcal{T}(p)$, and the tensor field $S$ on $\mathcal{T}(p)$ given by

$$
2\left\langle S_{X} Y, Z\right\rangle=\langle[X, Y], Z\rangle-\langle[X, Z], Y\rangle-\langle[Y, Z], X\rangle,
$$

for $X, Y, Z \in \mathfrak{t}(p)$. Let $\nabla$ be the Levi-Civita connection of $(\mathcal{T}(p),\langle\rangle$,$) . Then$ $\widetilde{\nabla}=\nabla-S$ is the connection on the Lie group $\mathcal{T}(p)$ for which every leftinvariant vector field is parallel. Thus, conditions (2.3) are satisfied and $S$ is a homogeneous quaternionic Kähler structure. Moreover, the holonomy algebra of the connection $\widetilde{\nabla}$ is trivial, and then $S$ provides the description of $\mathcal{T}(p)$ as a Lie group (see [19, p. 32, Eqs. (1.79)]).

Since $[\mathfrak{u}, \mathfrak{u}] \subset \mathfrak{u},[\mathfrak{u}, \tilde{\mathfrak{u}}] \subset \tilde{\mathfrak{u}},[\tilde{\mathfrak{u}}, \tilde{\mathfrak{u}}] \subset \mathfrak{u}$, and $\mathfrak{u}$ and $\tilde{\mathfrak{u}}$ are orthogonal, from (3.3) we have

$$
S_{U V \widetilde{W}}=0, \quad S_{\widetilde{U} V W}=0, \quad S_{U \widetilde{V} W}=0, \quad S_{\widetilde{U} \widetilde{W}}=0 .
$$


Table 2: The Q-representation $T: \mathfrak{u} \rightarrow \operatorname{End}(\tilde{\mathfrak{u}})$

\begin{tabular}{lcccc}
\hline & $\widetilde{G}_{0}$ & $\widetilde{H}_{0}$ & $\widetilde{G}_{1}$ & $\widetilde{H}_{1}$ \\
\hline$G_{0}$ & 0 & 0 & 0 & 0 \\
$H_{0}$ & $\frac{1}{2} \widetilde{G}_{0}$ & $\frac{1}{2} \widetilde{H}_{0}$ & $\frac{1}{2} \widetilde{G}_{1}$ & $\frac{1}{2} \widetilde{H}_{1}$ \\
$G_{1}$ & $\frac{-1}{2}\left(\widetilde{H}_{0}+\widetilde{H}_{1}\right)$ & $\frac{1}{2}\left(\widetilde{G}_{0}+\widetilde{G}_{1}\right)$ & $\frac{1}{2}\left(\widetilde{H}_{0}+\widetilde{H}_{1}\right)$ & $\frac{-1}{2}\left(\widetilde{G}_{0}+\widetilde{G}_{1}\right)$ \\
$H_{1}$ & $\frac{1}{2} \widetilde{G}_{1}$ & $\frac{1}{2} \widetilde{H}_{1}$ & $\frac{1}{2} \widetilde{G}_{0}$ & $\frac{1}{2} \widetilde{H}_{0}$ \\
$G_{2}$ & $\frac{-1}{2}\left(\sqrt{2} \widetilde{H}_{0}+t H_{2}\right)$ & $\frac{1}{2}\left(\sqrt{2} \widetilde{G}_{0}+\widetilde{G}_{2}\right)$ & $\frac{-1}{2}\left(\sqrt{2} \widetilde{H}_{1}+\widetilde{H}_{2}\right)$ & $\frac{1}{2}\left(\sqrt{2} \widetilde{G}_{1}-\widetilde{G}_{2}\right)$ \\
$H_{2}$ & $\frac{1}{2} \widetilde{G}_{2}$ & $\frac{1}{2} \widetilde{H}_{2}$ & $\frac{-1}{2} \widetilde{G}_{2}$ & $\frac{1}{2} \widetilde{H}_{2}$ \\
$X_{j+}$ & $\frac{1}{2} \widetilde{X}_{j}$ & $\frac{1}{2} \widetilde{X}_{j}$ & $\frac{-1}{2} \widetilde{X}_{j}$ & $\frac{-1}{2} \widetilde{X}_{j}$ \\
$X_{j-}$ & $\frac{-1}{2} \widetilde{X}_{j}$ & $\frac{1}{2} \widetilde{X}_{j}$ & $\frac{1}{2} \widetilde{X}_{j}$ & $\frac{-1}{2} \widetilde{X}_{j}$ \\
\hline & $\widetilde{G}_{2}$ & $\widetilde{H}_{2}$ & $\widetilde{X}_{i+}$ & $\widetilde{X}_{i-}$ \\
\hline$G_{0}$ & 0 & 0 & 0 & 0 \\
$H_{0}$ & $\frac{1}{2} \widetilde{G}_{2}$ & $\frac{1}{2} \widetilde{H}_{2}$ & $\frac{1}{2} \widetilde{X}_{i+}$ & $\frac{1}{2} \widetilde{X}_{i-}$ \\
$G_{1}$ & $-\widetilde{H}_{2}$ & 0 & 0 & 0 \\
$H_{1}$ & $\frac{-1}{2} \widetilde{G}_{2}$ & $\frac{1}{2} \widetilde{H}_{2}$ & 0 & 0 \\
$G_{2}$ & $\frac{1}{2}\left(\widetilde{H}_{0}-\widetilde{H}_{1}\right)$ & $\frac{-1}{2}\left(\widetilde{G}_{0}+\widetilde{G}_{1}\right)$ & 0 & $-\frac{\sqrt{2}}{2} \widetilde{X}_{i_{+}}$ \\
$H_{2}$ & $\frac{1}{2}\left(\widetilde{G}_{0}-\widetilde{G}_{1}\right)$ & $\frac{1}{2}\left(\widetilde{H}_{0}+\widetilde{H}_{1}\right)$ & $\frac{\sqrt{2}}{4} \widetilde{X}_{i+}$ & $\frac{-\sqrt{2}}{4} \widetilde{X}_{i}$ \\
$X_{j+}$ & $\frac{-\sqrt{2}}{2} \widetilde{X}_{j}$ & 0 & $\frac{\delta_{i j}}{2}\left(\widetilde{H}_{0}+\widetilde{H}_{1}+\sqrt{2} \widetilde{H}_{2}\right)$ & $\frac{\delta_{i j}}{2}\left(\widetilde{G}_{0}+\widetilde{G}_{1}\right)$ \\
$X_{j-}$ & $\frac{-\sqrt{2}}{2} \widetilde{X}_{j}$ & 0 & $\frac{-\delta_{i j}}{2}\left(\widetilde{G}_{0}+\widetilde{G}_{1}\right)$ & $\frac{\delta_{i j}}{2}\left(\widetilde{H}_{0}+\widetilde{H}_{1}-\sqrt{2} \widetilde{H}_{2}\right)$ \\
\hline \multicolumn{5}{c}{} \\
\hline
\end{tabular}

We have $\mathfrak{t}(p)^{*}=\mathfrak{u}^{*}+\tilde{\mathfrak{u}}^{*}$. Let

$$
\mathcal{B}^{*}=\left\{\gamma^{j}, \eta^{j}, \xi^{i+}, \xi^{i-}, \tilde{\gamma}^{j}, \tilde{\eta}^{j}, \tilde{\xi}^{i+}, \tilde{\xi}^{i-}: 0 \leq j \leq 2,1 \leq i \leq p\right\}
$$

be the basis of $\mathfrak{t}(p)^{*}$ dual to the basis $\mathcal{B}=\left\{G_{j}, H_{j}, X_{i+}, X_{i-}, \widetilde{G}_{j}, \widetilde{H}_{j}, \widetilde{X}_{i_{+}}, \widetilde{X}_{i_{-}}\right\}$ of $\mathfrak{t}(p)$. Then, if we denote by $S_{X}$ the 2 -form defined by $S_{X}(Y, Z)=S_{X Y Z}$, we have

Theorem 3.1. The homogeneous quaternionic Kähler structure $S$ on each rankthree Alekseevsky space $\mathcal{T}(p), p \geq 0$, which gives its description as the simplyconnected solvable Lie group with Lie algebra $\mathfrak{t}(p)$, is given, in terms of the basis $\mathcal{B}^{*}$ of $\mathfrak{t}(p)^{*}$, by

$$
\begin{aligned}
S_{\mid \mathfrak{u}^{*} \otimes \wedge^{2} \mathfrak{u}^{*}} & =\gamma^{0} \otimes\left(\gamma^{0} \wedge \eta^{0}\right)+\gamma^{1} \otimes\left(\gamma^{1} \wedge \eta^{1}\right) \\
& +\frac{\sqrt{2}}{2} \gamma^{2} \otimes\left(\gamma^{2} \wedge \eta^{2}\right)-\frac{1}{2}\left(\gamma^{1}+\frac{\sqrt{2}}{2} \gamma^{2}\right) \otimes \sum_{i}\left(\xi^{i+} \wedge \xi^{i-}\right) \\
& +\frac{1}{2} \sum_{i}\left(\xi^{i+} \otimes\left(\xi^{i-} \wedge\left(\gamma^{1}+\frac{\sqrt{2}}{2} \gamma^{2}\right)\right)+\xi^{i+} \otimes\left(\xi^{i+} \wedge\left(\eta^{1}+\frac{\sqrt{2}}{2} \eta^{2}\right)\right)\right. \\
& \left.\quad-\xi^{i-} \otimes\left(\xi^{i+} \wedge\left(\gamma^{1}-\frac{\sqrt{2}}{2} \gamma^{2}\right)\right)+\xi^{i-} \otimes\left(\xi^{i-} \wedge\left(\eta^{1}-\frac{\sqrt{2}}{2} \eta^{2}\right)\right)\right)
\end{aligned}
$$




$$
\begin{aligned}
& S_{\mid \mathfrak{u}^{*} \otimes \wedge^{2} \tilde{\mathfrak{u}}^{*}}=\frac{1}{2}\left(\gamma^{0}-\gamma^{1}-\sqrt{2} \gamma^{2}\right) \otimes\left(\tilde{\gamma}^{0} \wedge \tilde{\eta}^{0}\right)-\frac{1}{2}\left(\gamma^{0}-\gamma^{1}+\sqrt{2} \gamma^{2}\right) \otimes\left(\tilde{\gamma}^{1} \wedge \tilde{\eta}^{1}\right) \\
& -\frac{1}{2}\left(\gamma^{0}+\gamma^{1}\right) \otimes\left(\tilde{\gamma}^{2} \wedge \tilde{\eta}^{2}\right)+\frac{1}{2}\left(\gamma^{0}+\frac{\sqrt{2}}{2} \gamma^{2}\right) \otimes \sum_{i}\left(\tilde{\xi}^{i+} \wedge \tilde{\xi}^{i-}\right) \\
& +\frac{1}{2} \sum_{i}\left(\xi^{i+} \otimes\left(\tilde{\xi}^{i-} \wedge\left(\tilde{\gamma}^{1}+\frac{\sqrt{2}}{2} \tilde{\gamma}^{2}\right)\right)+\xi^{i+} \otimes\left(\tilde{\xi}^{i+} \wedge\left(\tilde{\eta}^{1}+\frac{\sqrt{2}}{2} \tilde{\eta}^{2}\right)\right)\right. \\
& \left.-\xi^{i-} \otimes\left(\tilde{\xi}^{i+} \wedge\left(\tilde{\gamma}^{1}-\frac{\sqrt{2}}{2} \tilde{\gamma}^{2}\right)\right)+\xi^{i-} \otimes\left(\tilde{\xi}^{i-} \wedge\left(\tilde{\eta}^{1}-\frac{\sqrt{2}}{2} \tilde{\eta}^{2}\right)\right)\right), \\
& S_{\tilde{G}_{0}}=\frac{1}{2}\left(\sum_{j=0}^{2}\left(\tilde{\gamma}^{j} \wedge \eta^{j}-\tilde{\eta}^{j} \wedge \gamma^{j}\right)+\sum_{i}\left(\tilde{\xi}^{i-} \wedge \xi^{i+}-\tilde{\xi}^{i+} \wedge \xi^{i-}\right)\right), \\
& S_{\tilde{H}_{0}}=\frac{1}{2}\left(\sum_{j=0}^{2}\left(\tilde{\gamma}^{j} \wedge \gamma^{j}+\tilde{\eta}^{j} \wedge \eta^{j}\right)+\sum_{i}\left(\tilde{\xi}^{i+} \wedge \xi^{i+}+\tilde{\xi}^{i-} \wedge \xi^{i-}\right)\right), \\
& S_{\tilde{G}_{1}}=\frac{1}{2}\left(\tilde{\gamma}^{0} \wedge \eta^{1}+\tilde{\eta}^{0} \wedge \gamma^{1}+\tilde{\gamma}^{1} \wedge \eta^{0}+\tilde{\eta}^{1} \wedge \gamma^{0}-\tilde{\gamma}^{2} \wedge \eta^{2}-\tilde{\eta}^{2} \wedge \gamma^{2}\right), \\
& S_{\tilde{H}_{1}}=\frac{1}{2}\left(-\tilde{\gamma}^{0} \wedge \gamma^{1}+\tilde{\eta}^{0} \wedge \eta^{1}-\tilde{\gamma}^{1} \wedge \gamma^{0}+\tilde{\eta}^{1} \wedge \eta^{0}-\tilde{\gamma}^{2} \wedge \gamma^{2}+\tilde{\eta}^{2} \wedge \eta^{2}\right), \\
& S_{\tilde{G}_{2}}=\frac{1}{2}\left(\left(\tilde{\gamma}^{0}-\tilde{\gamma}^{1}\right) \wedge \eta^{2}+\left(\tilde{\eta}^{0}-\tilde{\eta}^{1}\right) \wedge \gamma^{2}+\tilde{\gamma}^{2} \wedge\left(\eta^{0}-\eta^{1}\right)+\tilde{\eta}^{2} \wedge\left(\gamma^{0}-\gamma^{1}\right)\right) \\
& -\frac{\sqrt{2}}{4} \sum_{i}\left(\tilde{\xi}^{i+} \wedge \xi^{i-}+\tilde{\xi}^{i-} \wedge \xi^{i+}\right), \\
& S_{\tilde{H}_{2}}=\frac{1}{2}\left(-\left(\tilde{\gamma}^{0}+\tilde{\gamma}^{1}\right) \wedge \gamma^{2}+\left(\tilde{\eta}^{0}+\tilde{\eta}^{1}\right) \wedge \eta^{2}-\tilde{\gamma}^{2} \wedge\left(\gamma^{0}+\gamma^{1}\right)+\tilde{\eta}^{2} \wedge\left(\eta^{0}+\eta^{1}\right)\right) \\
& +\frac{\sqrt{2}}{4} \sum_{i}\left(\tilde{\xi}^{i+} \wedge \xi^{i+}-\tilde{\xi}^{i-} \wedge \xi^{i-}\right), \\
& S_{\widetilde{X}_{i+}}=\frac{1}{2}\left(-\tilde{\gamma}^{0} \wedge \xi^{i-}+\tilde{\eta}^{0} \wedge \xi^{i+}\right)+\frac{\sqrt{2}}{4}\left(-\tilde{\gamma}^{2} \wedge \xi^{i-}+\tilde{\eta}^{2} \wedge \xi^{i+}\right) \\
& +\frac{1}{2}\left(\tilde{\xi}^{i+} \wedge\left(\eta^{0}+\frac{\sqrt{2}}{2} \eta^{2}\right)-\tilde{\xi}^{i-} \wedge\left(\gamma^{0}+\frac{\sqrt{2}}{2} \gamma^{2}\right)\right), \\
& S_{\widetilde{X}_{i_{-}}}=\frac{1}{2}\left(\tilde{\gamma}^{0} \wedge \xi^{i+}+\tilde{\eta}^{0} \wedge \xi^{i-}\right)-\frac{\sqrt{2}}{4}\left(\tilde{\gamma}^{2} \wedge \xi^{i+}+\tilde{\eta}^{2} \wedge \xi^{i-}\right) \\
& +\frac{1}{2}\left(\tilde{\xi}^{i+} \wedge\left(\gamma^{0}-\frac{\sqrt{2}}{2} \gamma^{2}\right)+\tilde{\xi}^{i-} \wedge\left(\eta^{0}-\frac{\sqrt{2}}{2} \eta^{2}\right)\right) .
\end{aligned}
$$

Proof. Let $U, V, W \in \mathfrak{u}$. By (3.3),

$$
S_{U V W}=\frac{1}{2}(\langle[U, V], W\rangle-\langle[U, W], V\rangle-\langle[V, W], U)\rangle .
$$

On account of (3.1), and by the equation $S_{U V \widetilde{W}}=0$ in (3.4), from (3.5) one obtains the values of $S_{U V W}$ for $U, V$ and $W$ in the orthonormal basis $\mathcal{B}$, and hence we get the expression of $S_{\mid \mathfrak{u}^{*} \otimes \wedge^{2} \mathfrak{u}^{*}}$. In order to get $S_{\mid \mathfrak{u}^{*} \otimes \wedge^{2} \tilde{\mathfrak{u}}^{*}}$, we use (3.3), (3.2), the equation $S_{U \tilde{V} W}=0$ in (3.4) and Table 2, since $[U, \widetilde{V}]=$ $T_{U} \widetilde{V}$. Now, from (3.3), by using (3.2), Table 2, and the equations $S_{\widetilde{U} V W}=$ $S_{\tilde{U} \widetilde{V} \widetilde{W}}=0$ in (3.4), we get $S_{\widetilde{U}}$ for each $\widetilde{U}=\widetilde{G}_{j}, \widetilde{H}_{j}, \widetilde{X}_{i_{+}}, \widetilde{X}_{i_{-}}$.

\section{Types of homogeneous quaternionic Kähler structures on the spaces $\mathcal{T}(p)$}

We now determine the type of the previously obtained structure $S$ on each $\mathcal{T}(p)$.

To begin with, from the expression of $S$ in Theorem 3.1 and from Table 1 we 
get

Lemma 4.1. The forms $\theta^{a}, a=1,2,3$, in (2.5) corresponding to the homogeneous quaternionic Kähler structure $S$ in Theorem 3.1 are given by

$$
\theta^{1}=-\frac{1}{2}\left(\gamma^{0}+\gamma^{1}+\sqrt{2} \gamma^{2}\right), \quad \theta^{2}=-\tilde{\eta}^{0}, \quad \theta^{3}=\tilde{\gamma}^{0}
$$
have

We have $S=\Theta+\mathcal{A}$, where $\Theta$ is given by (2.6). Then, by Lemma 4.1 we

$$
\begin{gathered}
\Theta_{G_{0} Y Z}=\Theta_{G_{1} Y Z}=-\frac{1}{4}\left\langle J_{1} Y, Z\right\rangle, \quad \Theta_{G_{2} Y Z}=-\frac{\sqrt{2}}{4}\left\langle J_{1} Y, Z\right\rangle \\
\Theta_{\widetilde{G}_{0} Y Z}=\frac{1}{2}\left\langle J_{3} Y, Z\right\rangle, \quad \Theta_{\widetilde{H}_{0} Y Z}=-\frac{1}{2}\left\langle J_{2} Y, Z\right\rangle,
\end{gathered}
$$

and $\Theta_{H_{j}}=\Theta_{\widetilde{G}_{1}}=\Theta_{\widetilde{G}_{2}}=\Theta_{\widetilde{H}_{1}}=\Theta_{\widetilde{H}_{2}}=\Theta_{X_{i+}}=\Theta_{X_{i-}}=\Theta_{\widetilde{X}_{i_{+}}}=\Theta_{\widetilde{X}_{i_{-}}}=0$, for $0 \leq j \leq 2$ and $1 \leq i \leq p$, where $\Theta_{X}(Y, Z)=\Theta_{X Y Z}$. Then, by using Table 1 , we obtain

Proposition 4.1. The tensor field $\Theta$ on $\mathcal{T}(p)$ corresponding to the homogeneous quaternionic Kähler structure $S$ in Theorem 3.1 is given by

$$
\begin{aligned}
& \Theta_{G_{0}}=\frac{1}{4}\left(\sum_{j=0}^{2}\left(\gamma^{j} \wedge \eta^{j}-\tilde{\gamma}^{j} \wedge \tilde{\eta}^{j}\right)+\sum_{i}\left(\tilde{\xi}^{i+} \wedge \tilde{\xi}^{i-}-\xi^{i+} \wedge \xi^{i-}\right)\right), \\
& \Theta_{\widetilde{G}_{0}}=\frac{1}{2}\left(\sum_{j=0}^{2}\left(\gamma^{j} \wedge \tilde{\eta}^{j}-\eta^{j} \wedge \tilde{\gamma}^{j}\right)+\sum_{i}\left(\xi^{i-} \wedge \tilde{\xi}^{i+}-\xi^{i+} \wedge \tilde{\xi}^{i-}\right)\right), \\
& \Theta_{\widetilde{H}_{0}}=-\frac{1}{2}\left(\sum_{j=0}^{2}\left(\gamma^{j} \wedge \tilde{\gamma}^{j}+\eta^{j} \wedge \tilde{\eta}^{j}\right)+\sum_{i}\left(\xi^{i+} \wedge \tilde{\xi}^{i+}+\xi^{i-} \wedge \tilde{\xi}^{i-}\right)\right), \\
& \Theta_{G_{1}}=\Theta_{G_{0}}, \quad \Theta_{G_{2}}=\sqrt{2} \Theta_{G_{0}}, \\
& \Theta_{H_{j}}=\Theta_{\widetilde{G}_{1}}=\Theta_{\widetilde{G}_{2}}=\Theta_{\widetilde{H}_{1}}=\Theta_{\widetilde{H}_{2}}=\Theta_{X_{i+}}=\Theta_{X_{i-}}=\Theta_{\widetilde{X}_{i+}}=\Theta_{\widetilde{X}_{i_{-}}}=0, \\
& 0 \leq j \leq 2,1 \leq i \leq p .
\end{aligned}
$$

We then have

Theorem 4.1. The homogeneous quaternionic Kähler structure on each rankthree Alekseevsky space $\mathcal{T}(p), p \geq 0$, given in Theorem 3.1, has a nonzero component in $\mathcal{Q} \mathcal{K}_{i}$, for $i=1, \ldots, 5$.

Proof. The structure is given as $S=\Theta+\mathcal{A}$, and the values of the 1 -forms $\theta^{a}$ are given in Lemma 4.1. Then we firstly have that, as for instance,

$$
\sum_{a=1}^{3} \theta^{a}\left(J_{a} H_{1}\right)=-\frac{1}{2} \neq 0
$$

the component $\Theta$ of the structure $S$ does not belong to $\mathcal{Q} \mathcal{K}_{2}$.

Moreover, from Proposition 4.1, one has that the nonzero values of $\Theta_{X Y Z}$ are those with

$$
X=G_{0}, G_{1}, G_{2}, \widetilde{G}_{0}, \widetilde{H}_{0}
$$


In particular one has the next nonzero values of $\Theta_{X X Y}$.

$$
\begin{gathered}
\Theta_{G_{0} G_{0} H_{0}}=\Theta_{G_{1} G_{1} H_{1}}=\frac{1}{4}, \quad \Theta_{G_{2} G_{2} H_{2}}=\frac{\sqrt{2}}{4}, \\
\Theta_{\widetilde{G}_{0} \widetilde{G}_{0} H_{0}}=\Theta_{\widetilde{H}_{0} \widetilde{H}_{0} H_{0}}=\frac{1}{2} .
\end{gathered}
$$

Suppose that $\Theta \in \mathcal{Q} \mathcal{K}_{1}$, so there would be a 1-form $\theta$ as that in Theorem 2.1, and in particular we would have

$$
\frac{1}{4}=\Theta_{G_{0} G_{0} H_{0}}=\theta\left(H_{0}\right), \quad \frac{1}{2}=\Theta_{\widetilde{G}_{0} \widetilde{G}_{0} H_{0}}=\theta\left(H_{0}\right),
$$

which is absurd. Whence $\Theta \in \mathcal{Q K}_{12} \backslash\left\{\mathcal{Q K}_{1} \cup \mathcal{Q} \mathcal{K}_{2}\right\}$.

Further, as $\operatorname{dim} \mathcal{T}(p)=12+4 p$ and on account of (4.1), the form $\vartheta$ defining the $\mathcal{Q K}_{3}$-component (see Theorem 2.1), that is,

$$
\vartheta=\frac{1}{2+\operatorname{dim} \mathfrak{t}(p)} c_{12}(\mathcal{A})=\frac{1}{14+4 p} c_{12}(\mathcal{A})
$$

is given by

$$
\vartheta=\frac{1}{8(7+2 p)}\left\langle(11+4 p) H_{0}+(3+4 p) H_{1}+\sqrt{2} H_{2}, \cdot\right\rangle .
$$

Hence the structure $S$ has nonzero component in $\mathcal{Q K}_{3}$ for any $p \geq 0$.

Consider now the operator $\Phi: \widehat{\mathcal{V}} \rightarrow \widehat{\mathcal{V}}$ defined by

$$
\Phi(\mathcal{A})_{X Y Z}=\mathcal{A}_{Y Z X}+\mathcal{A}_{Z X Y}+\sum_{a=1}^{3}\left(\mathcal{A}_{J_{a} Y J_{a} Z X}+\mathcal{A}_{J_{a} Z X J_{a} Y}\right)
$$

having eigenvalues 2 and -4 , with corresponding eigenspaces $\mathcal{Q} \mathcal{K}_{34}$ and $\mathcal{Q} \mathcal{K}_{5}$, respectively (see Theorem 2.1). Consider $\mathcal{A}^{\vartheta} \in \mathcal{Q} \mathcal{K}_{3}$, given by

$$
\mathcal{A}_{X Y Z}^{\vartheta}=\langle X, Y\rangle \vartheta(Z)-\langle X, Z\rangle \vartheta(Y)+\sum_{a=1}^{3}\left(\left\langle X, J_{a} Y\right\rangle \vartheta\left(J_{a} Z\right)-\left\langle X, J_{a} Z\right\rangle \vartheta\left(J_{a} Y\right)\right),
$$

where $\vartheta$ stands for the 1 -form (4.2). Then $\mathcal{A}-\mathcal{A}^{\vartheta} \in \mathcal{Q K} \mathcal{K}_{45}$, so that

$$
\Phi\left(\mathcal{A}-\mathcal{A}^{\vartheta}\right)_{X Y Z}=\Phi(\mathcal{A})_{X Y Z}-2 \mathcal{A}_{X Y Z}^{\vartheta} .
$$

Taking then for instance the vectors $X=Y=G_{0}, Z=H_{0}$, we get

$$
\left(\mathcal{A}-\mathcal{A}^{\vartheta}\right)_{G_{0} G_{0} H_{0}}=\frac{5+p}{2(7+2 p)}, \quad \Phi\left(\mathcal{A}-\mathcal{A}^{\vartheta}\right)_{G_{0} G_{0} H_{0}}=-\frac{16+5 p}{7+2 p},
$$

hence $\mathcal{A}-\mathcal{A}^{\vartheta} \in \mathcal{Q K}_{45} \backslash\left\{\mathcal{Q K} \mathcal{K}_{4} \cup \mathcal{Q} \mathcal{K}_{5}\right\}$ for all $p \geq 0$. That is, $S$ has a nonzero component in each basic type. 


\section{References}

[1] D. V. Alekseevsky, Compact quaternion spaces, Funct. Anal. Appl. 2 (1968), 106114 .

[2] D. V. Alekseevsky, Classification of quaternionic spaces with a transitive solvable group of motions, Math. USSR Isvestija 9 (1975), 297-339.

[3] D. V. Alekseevsky and V. Cortés, Isometry groups of homogeneous quaternionic Kähler manifolds, J. Geom. Anal. 9 (1999), no. 4, 513-545.

[4] W. Ambrose and I. M. Singer, On homogeneous Riemannian manifolds, Duke Math. J. 25 (1958), 647-669.

[5] J. Bagger and E. Witten, Matter couplings in $N=2$ supergravity, Nucl. Phys. B 222 (1983), 1-10.

[6] A. L. Besse, Einstein manifolds, Mathematik und ihrer Grenzgebiete, 3. Folge, vol. 10, Springer, Berlin, Heidelberg and New York, 1987.

[7] M. Castrillón López, P. M. Gadea, and J. A. Oubiña, Homogeneous quaternionic Kähler structures on eight-dimensional non-compact quaternion-Kähler symmetric spaces, Math. Phys. Anal. Geom. 12 (2009), no. 1, 47-74.

[8] M. Castrillón López, P. M. Gadea, and J. A. Oubiña, Homogeneous quaternionic Kähler structures on 12-dimensional Alekseevsky spaces, J. Geom. Phys. 57 (2007), no. 2, 2098-2113.

[9] M. Castrillón López, P. M. Gadea, and A. F. Swann, Homogeneous quaternionic Kähler structures and quaternionic hyperbolic space, Transform. Groups 11 (2006), no. 4, 575-608.

[10] S. Cecotti, Homogeneous Kähler manifolds and T-algebras in $N=2$ supergravity and superstrings, Comm. Math. Phys. 124 (1989), no. 1, 23-55.

[11] V. Cortés, Alekseevskian spaces, Differential Geom. Appl. 6 (1996), no. 2, 129168.

[12] A. Fino, Intrinsic torsion and weak holonomy, Math. J. Toyama Univ. 21 (1998), $1-22$.

[13] N. J. Hitchin, Kählerian twistor spaces, Proc. London Math. Soc. (3) 43 (1981), no. $1,133-150$.

[14] S. Ishihara, Quaternion Kählerian manifolds, J. Differential Geom. 9 (1974), 483500.

[15] V. F. Kiričenko, On homogeneous Riemannian spaces with an invariant structure tensor, Dokl. Akad. Nauk SSSR 252 (1980), no. 2, 291-293.

[16] C. LeBrun and S. M. Salamon, Strong rigidity of positive quaternion-Kähler manifolds, Inv. Math. 118 (1994), 109-132.

[17] Y. S. Poon and S. M. Salamon, Quaternionic Kähler 8-manifolds with positive scalar curvature, J. Differential Geom. 33 (1991), 363-378.

[18] A. F. Swann, HyperKähler and quaternionic Kähler geometry, Math. Ann. 289 (1991), 421-450.

[19] F. Tricerri and L. Vanhecke, Homogeneous structures on Riemannian manifolds, London Math. Soc. Lect. Notes Ser. vol. 83, Cambridge Univ. Press, 1983. 
[20] B. De Wit and A. van Proeyen, Special geometry, cubic polynomials and homogeneous quaternionic spaces, Comm. Math. Phys. 149 (1992), no. 2, 307-333.

[21] J. A. Wolf, Complex homogeneous contact manifolds and quaternionic symmetric spaces, J. Math. Mech. 14 (1965), 1033-1047.

\section{Authors' addresses:}

W.B.: École Normale Supérieure d'Enseignement Technologique d'Oran, Département de Mathématiques et Informatique, B.P. 1523, El M’Naouar, Oran, Algeria. E-mail: wafa.batat@enset-oran.dz

P.M.G.: Instituto de Física Fundamental, CSIC, Serrano 113-bis, 28006-Madrid, Spain. E-mail: pmgadea@iec.csic.es

J.A.O.: Departamento de Xeometría e Topoloxía, Facultade de Matemáticas, Universidade de Santiago de Compostela, 15782-Santiago de Compostela, Spain. Corresponding author, Tl. +34881813141, Fax +34981597054, E-mail: ja.oubina@usc.es 